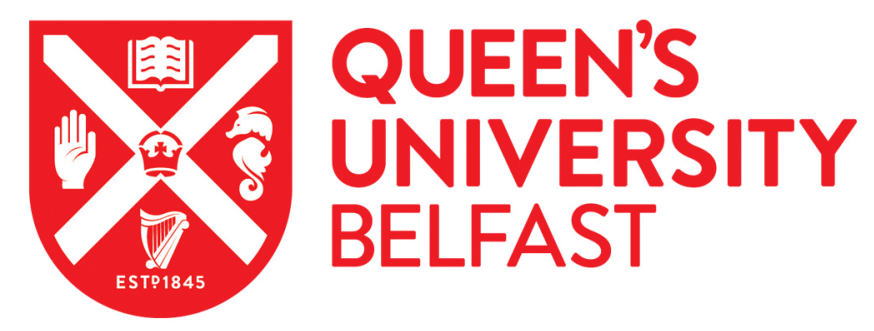

\title{
Electronically Reconfigurable Liquid Crystal Based Mm-Wave Polarization Converter
}

Doumanis, E., Goussetis, G., Dickie, R., Cahill, R., Baine, P., Bain, M., Fusco, V., Encinar, J. A., \& Toso, G. (2014). Electronically Reconfigurable Liquid Crystal Based Mm-Wave Polarization Converter. IEEE Transactions on Antennas and Propagation, 62(4), 2302-2307. https://doi.org/10.1109/TAP.2014.2302844

Published in:

IEEE Transactions on Antennas and Propagation

Document Version:

Peer reviewed version

Queen's University Belfast - Research Portal:

Link to publication record in Queen's University Belfast Research Portal

\begin{abstract}
Publisher rights
(c) 2014 IEEE. Personal use of this material is permitted. Permission from IEEE must be obtained for all other uses, in any current or future media, including

reprinting/republishing this material for advertising or promotional purposes, creating new collective works, for resale or redistribution to servers or lists, or reuse of any copyrighted component of this work in other works.
\end{abstract}

\section{General rights}

Copyright for the publications made accessible via the Queen's University Belfast Research Portal is retained by the author(s) and / or other copyright owners and it is a condition of accessing these publications that users recognise and abide by the legal requirements associated with these rights.

\section{Take down policy}

The Research Portal is Queen's institutional repository that provides access to Queen's research output. Every effort has been made to ensure that content in the Research Portal does not infringe any person's rights, or applicable UK laws. If you discover content in the Research Portal that you believe breaches copyright or violates any law, please contact openaccess@qub.ac.uk. 


\section{Electronically Reconfigurable Liquid Crystal Based Mm-wave Polarization Converter}

\author{
E. Doumanis, Member, IEEE, G. Goussetis, Senior \\ Member, IEEE, R. Dickie, R. Cahill Senior Member, \\ IEEE, P. Baine, M. Bain, V. Fusco, Fellow IEEE, J. A. \\ Encinar, Fellow IEEE, G. Toso Senior Member, IEEE
}

\begin{abstract}
An electronically tunable reflection polarizer which exploits the dielectric anisotropy of nematic liquid crystals (LC) has been designed, fabricated and measured in a frequency band centered at $130 \mathrm{GHz}$. The phase agile polarizing mirror converts an incident slant $45^{\circ}$ signal upon reflection to Right Hand Circular (RHCP), orthogonal linear $\left(-45^{\circ}\right)$ or Left Hand Circular (LHCP) polarization depending on the value of the voltage biasing the LC mixture. In the experimental set-up this is achieved by applying a low frequency bias voltage of $0 \mathrm{~V}, 40 \mathrm{~V}$ and $89 \mathrm{~V}$ respectively, across the cavity containing the LC material.
\end{abstract}

Index Terms - mm-wave, submm-wave, tunable, polarizer, liquid crystals, space communications, imaging, remote sensing, earth observation, polarimetric systems, interferometry.

\section{INTRODUCTION}

Satellite polarimetric imaging systems, such as those used for passive and active Earth observation to measure the surface wind vector from space $[1,2]$ and vegetation properties [3], commonly involve dedicated receive and transmit chains for each polarization state. Tunable polarizers can reduce redundancy, volume/mass budget and cost. Dynamic polarization agility is also desirable in radar applications for defense and remote sensing to enhance detection and measurement of a feature in a radar scene [4] as well as wireless and satellite telecommunications to minimize feed losses and polarization purity impairments. Polarization agility in quasi-optical mm-wave systems can also be used to create tunable isolators (switches) [5] as well as frequency tunable interferometers for filtering and diplexing [6].

Traditional tunable polarizer technology relies on mechanical motors or rotors [4] leading to increased energy consumption and mass as well as compromised reliability. In

This Manuscript received August 29, 2013. This work was supported by the European Space Agency, under project 4000103061. G. Goussetis would like to acknowledge support by FP7 project DORADA (IAPP-2013-610691).

E. Doumanis is with Bell-Labs Alcatel-Lucent, Blunchardstown Industrial Park, Dublin 15, Ireland (e-mail: efstratios.doumanis@alcatel-lucent.com)

G. Goussetis is with the School of Engineering and Physical Sciences, Heriot-Watt University, Edinburgh EH14 4AS, UK (e-mail: g.goussetis@ieee.org)

R. Dickie, R. Cahill, P. Baine, M. Bain and V. Fusco are with the Institute of Electronics, Communications and Information Technology, Queen's University Belfast, Belfast BT3 9DT, Northern Ireland, UK, (e-mail: r.cahill@qub.ac.uk)

J. A. Encinar is with the Department of Electromagnetism and Circuit Theory, Universidad Politécnica de Madrid, E-28040, Madrid, Spain (e-mail: jose.encinar@upm.es).

G. Toso is with the European Space Agency ESA-ESTEC, 2200 AG Noordwijk, The Netherlands, (e-mail: giovanni.toso@esa.int). order to address such limitations, integrated solutions based on e.g. MEMS [7] and piezoelectric ultrasonic motors [8] have been proposed. Despite their compact physical dimensions, these technologies are suitable for switched-based architectures offering discrete polarization states. Moreover, such technologies are mostly relevant to waveguide-based polarizers which are difficult to scale to (sub)mm-wave frequencies; free-space polarizers would typically require multiple actuators which is undesirable for practical implementation.

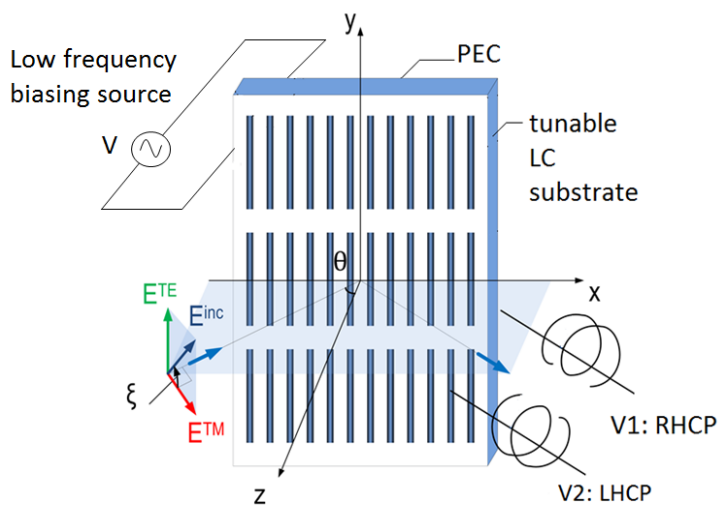

Fig. 1. Schematic of the proposed reconfigurable polarizer that exploits a tunable Liquid Crystal substrate biased by a low frequency quasi-static source.

Recently the use of nematic liquid crystals as a dielectric with electronically tunable permittivity has emerged as a technology suitable for continuous tuning of (sub)mm-wave and $\mathrm{THz}$ devices [9]. By applying an external field dynamic adjustment of the effective dielectric properties can be achieved [10], [11]. A waveguide-based liquid crystal polarizer was proposed in [12] as means to deliver continuous tuning of the polarization state between $-90^{\circ}$ to $90^{\circ}$ and was demonstrated at Q-band. Significantly, the cost and complexity of integrating liquid crystals in multi-wavelength free-space devices does not increase proportionally with the size; this integration is also compatible with mature semiconductor technologies hence enabling scaling to the (sub)mm-wave range. Liquid crystals have therefore been successfully employed in the development of tunable frequency selective surfaces [13]. They have also been employed in the development of tunable reflectarray cells for sum and difference monopulse radiation patterns [14] and dynamic beam steering at W-band [15].

In this communication we present a mm-wave tunable quasi-optical reflection polarizer that exploits liquid crystal technology to dynamically change the polarization state of an incoming wave. In particular we demonstrate the first reported free-space polarizer operating at frequencies larger than 100 $\mathrm{GHz}$ with the capability to convert a linearly polarized incoming wave to a reflected wave with a polarization state that can be selected to be RHCP, LHCP or the orthogonal linear polarization, thus covering an entire meridian on the Poincare sphere and, when considering the polarization of the incident signal, covers the four key canonical polarization 
states. The polarizer design is based on a recently proposed class of engineered mirrors [16]. A static W-band prototype has been presented in [17]. This feature is exploited here in conjunction with the tunable dielectric permittivity tensor of a liquid crystal mixture in order to develop an electronically reconfigurable reflection polarizer. A schematic of the structure is depicted in Fig 1.

\section{RECONFIGURABLE MM-WAVE POLARIZER}

\section{A. Design and realization}

The operation of the reconfigurable polarizer is demonstrated by means of a structure designed to operate at $130 \mathrm{GHz}$. Simulations of the electromagnetic scattering have been performed using the commercial electromagnetic solver CST [18]. Fig. 2 depicts the geometry and dimensions of a unit cell and a crossectional view of the device. The periodic array of dipoles is printed on a $100 \mathrm{~mm}$ diameter metal plated quartz substrate with dielectric permittivity $\varepsilon_{r}=3.78$ and loss $\operatorname{tangent} \tan \delta=0.0004$. The thickness of the metallized pattern is $3 \mu \mathrm{m}$. In order to reduce the possibility of breakage during processing, the thickness of the quartz substrate is chosen to be $250 \mu \mathrm{m}$ and a metalized silicon wafer is used to form the ground plane. A thin $(100 \mathrm{~nm})$ resistive boron diffused polycrystaline silicon film [19] was deposited by a low pressure Chemical Vapour Deposition process at $620^{\circ} \mathrm{C}$ on the un-patterned side of the quartz wafer to give an almost $\mathrm{mm}$ wave transparent but low frequency conductive layer. This layer is used for the establishment of a low frequency quasistatic electric field in the cavity to bias the liquid crystals; it has been experimentally determined that a low frequency field is best suitable for the biasing of the LC [13]. The average measured sheet resistance was $28 \mathrm{k} \Omega / \square$ and therefore this layer does not interact strongly $(<0.2 \mathrm{~dB}$ transmission loss $)$ with the incoming $\mathrm{mm}$-wave signal.

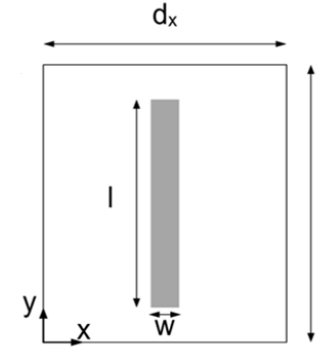

(a)

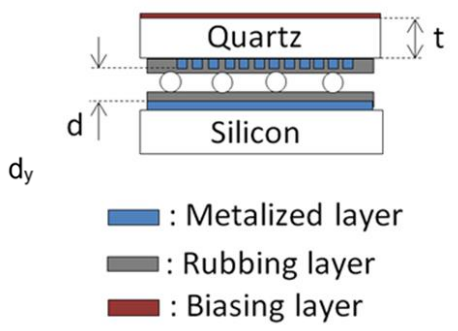

(b)
Fig. 2. Schematic of unit cell (a) top view and (b) cross-section of the polarizer. The circles depict the microsphere spacers that define the thickness of the cavity in which the liquid crystal mixture is encapsulated. Dimensions (in mm): $1=0.6, \mathrm{w}=0.04, \mathrm{dx}=0.8, \mathrm{dy}=1.0$, quartz thickness ( $\mathrm{t}$ ) $=0.25$, cavity thickness $(\mathrm{d})=0.05$.

In order to align the direction of the LC molecules parallel to the cell substrate, an alignment layer consisting of a $1 \mu \mathrm{m}$ thick polyimide film was deposited on the two metalized surfaces and subsequently rubbed with velvet to form microgrooves. The two wafers were separated by a $50 \mu \mathrm{m}$ gap, which was created by placing Micropearl SP precision glass spheres [20] around the outer region of the device, and bonded using slow drying glue, thus forming a cavity where the LC mixture is hosted. The construction of the polarizer was completed by applying a thin film of epoxy resin around the edge of the stacked wafers to seal the cavity leaving a small filling port for introducing the LC material. A vacuum filling technique was employed to insert the liquid crystals into the $50 \mu \mathrm{m}$ thick cavity via the filling port; the elongated LC molecules align along the groves of this rubbing layer during filling. The cavity was subsequently sealed and two small copper to aluminium contacts were attached on the outside of the cell using silver conductive glue to enable biasing. For the construction of this prototype device we have used GT3-23001, which is a custom LC mixture developed for microwave applications by Merck and characterised at $\mathrm{mm}$ wavelengths [19].

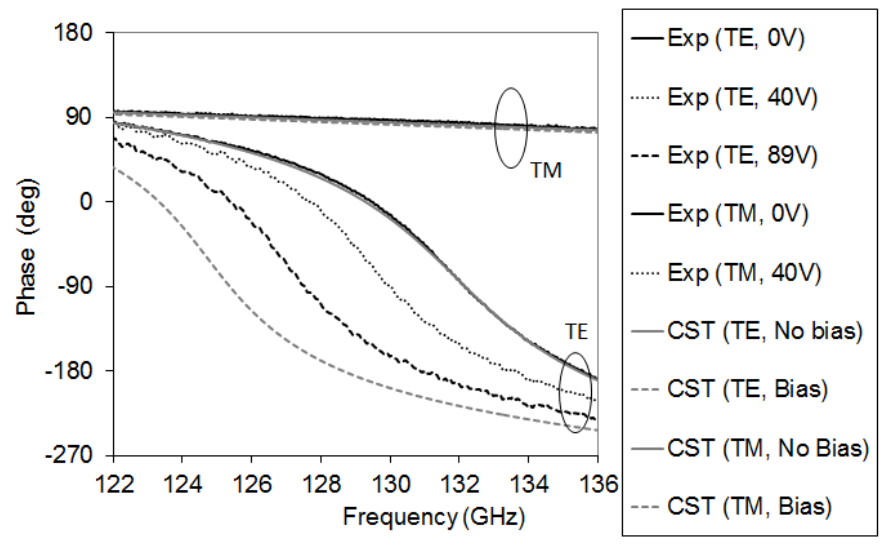

(a)

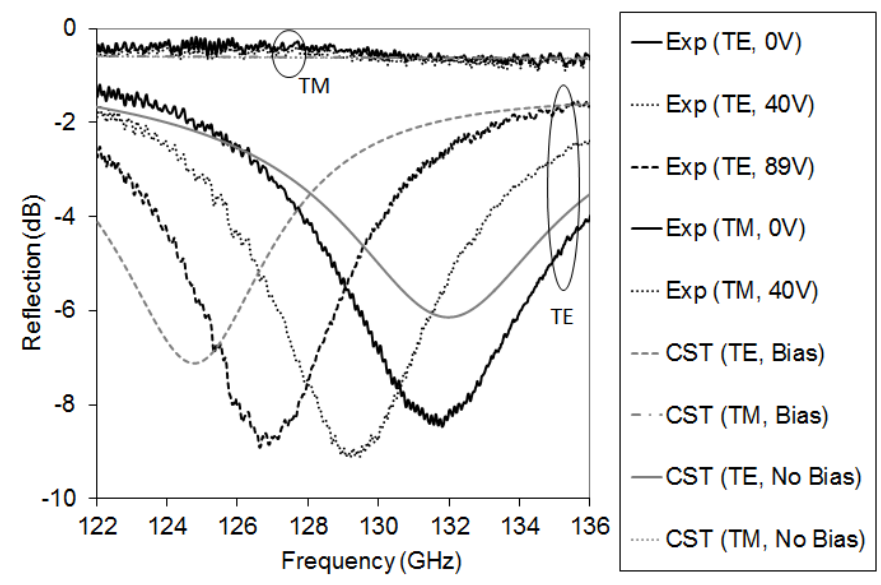

(b)

Fig. 3. Simulated reflection (a) phases and (b) magnitudes obtained with CST for TE and TM incident waves incident on the LC based polarizer shown in Fig. 2 at angle $\theta=45^{\circ}$. The tensors shown in eq. (1) have been used for the simulation of the unbiased (OFF) and biased (ON) states, where $\varepsilon_{/ /}=3.25$, $\tan \delta_{/ /}=0.015, \varepsilon_{\perp}=2.47, \tan \delta_{\perp}=0.02$. Measured reflection phases and amplitudes for various biasing voltages are superimposed.

The rubbing layers as well as the microsphere spacers have negligible effect on the electromagnetic response of the structure and therefore have not been included in simulation. Likewise dipole thickness has been ignored as it was shown 
that the performance for metal features of the order of $3 \mu \mathrm{m}$ is almost identical to that for 2D flat elements. As described in [21], the dielectric properties of the liquid crystal mixture are best characterized by a permittivity tensor that takes into account the orientation of the long molecules of the mixture. Assuming that the molecules are aligned along the y-axis in the off state (unbiased) and along the z-axis in the on state (biased) (Fig. 2), then the complex dielectric permittivities in those two cases are:

$$
\overline{\overline{\varepsilon_{O F F}}}=\left(\begin{array}{ccc}
\varepsilon_{\perp} & 0 & 0 \\
0 & \varepsilon_{\|} & 0 \\
0 & 0 & \varepsilon_{\perp}
\end{array}\right) \quad \overline{\overline{\varepsilon_{O N}}}=\left(\begin{array}{ccc}
\varepsilon_{\perp} & 0 & 0 \\
0 & \varepsilon_{\perp} & 0 \\
0 & 0 & \varepsilon_{\|}
\end{array}\right)
$$

Similar tensors describe the anisotropic loss tangent and are not presented here for brevity.

Based on these parameters and the aforementioned substrate stack, the dimensions of the dipole array were optimized using the design methodology described in [16]. The incidence angle, marked as $\theta$ in Fig. 1, is $45^{\circ}$. For the purposes of the design we have used the dielectric properties for GT3-23001 measured in the range $140-165 \mathrm{GHz}$ [19]: $\varepsilon_{r / /}=3.25$, $\tan \delta_{/ /}=0.015, \varepsilon_{r \perp}=2.47, \tan \delta_{\perp}=0.02$.

The primary design aim is to enable the polarization of the reflected waves to vary between RHCP and LHCP at the two extreme values of the permittivity tensor for zero, $\overline{\overline{\varepsilon_{O F F}}}$, and fully biased liquid crystals, $\overline{\overline{\varepsilon_{O N}}}$. With reference to the schematic shown in Fig. 1, the reflection properties of the TM polarized waves are negligibly affected by the dielectric properties of the liquid crystal; therefore the required range of reflection coefficient phases for TE polarized waves can be obtained. Given that between these two values, the polarization of the reflected waves moves continuously across an entire meridian of the Poincare sphere, linear polarized waves which are orientated orthogonal to the direction of the incidence signals will occur across this continuum; this will occur at the frequency where the phase difference between the TE and TM reflected field is $180^{\circ}$.

A further design consideration relates to the thermal losses. Structures such as the one shown in Fig. 1 are known to exhibit increased thermal losses around the frequency where the reflection phase is zero. This is due to a resonance formed in the cavity between the periodic array and the ground plane and is also known as the Artificial Magnetic Conductor (AMC) frequency [22]. As a general observation, array designs which provide a large change in the TE reflection phases for a given set of $\overline{\overline{\varepsilon_{O F F}}}$ and $\overline{\overline{\varepsilon_{O N}}}$ tensors, are also unavoidably subjected to increased losses near the resonance. A trade-off between the tuning range of the TE reflection phase and insertion loss is therefore required during the design stage.

The optimized dimensions for the dipole array are provided in the legend of Fig. 2 and the simulated reflection phase and magnitude coefficients of the polarizer in the unbiased $\left(\varepsilon_{r \perp}\right)$ and fully biased $\left(\varepsilon_{r / /}\right)$ states of the liquid crystal are depicted in Fig. 3. It is noted that in the phase responses shown in Fig. 3 the frequency of the reflection minimum observed in Fig. $3 \mathrm{~b}$ does not coincide with the zero reflection phase (Fig. 3a), as is commonly the case in AMCs [22]; instead an additional phase delay of approximately $90^{\circ}$ is introduced as a result of propagation within the substrate. This is attributed to the choice of the reference plane. It is noted that the reflection minimum still coincides with the maximum group delay (steepest reflection phase slope) as expected. The choice of the reference plane further explains the reflection phase values observed for TM polarized waves.

As shown in Fig. 3a, the TM reflection phase is scarcely affected by the biasing of the liquid crystal but for TE the reflection phase varies across a range of about $184^{\circ}$ at 130 $\mathrm{GHz}$. The cavity resonance associated with the zero reflection phase [22] is evident in Fig. 3b where the TE reflection coefficient is shown to be lower than the value predicted for the TM plane, peaking at $-6 \mathrm{~dB}$ and $-7 \mathrm{~dB}$ for the unbiased and biased case at the AMC frequency respectively. The relatively high level of losses for TE incidence waves can be understood in view of the discussion in reference [9], where it was elaborated that larger variations of the reflection phase for this class of devices is associated with increased losses.

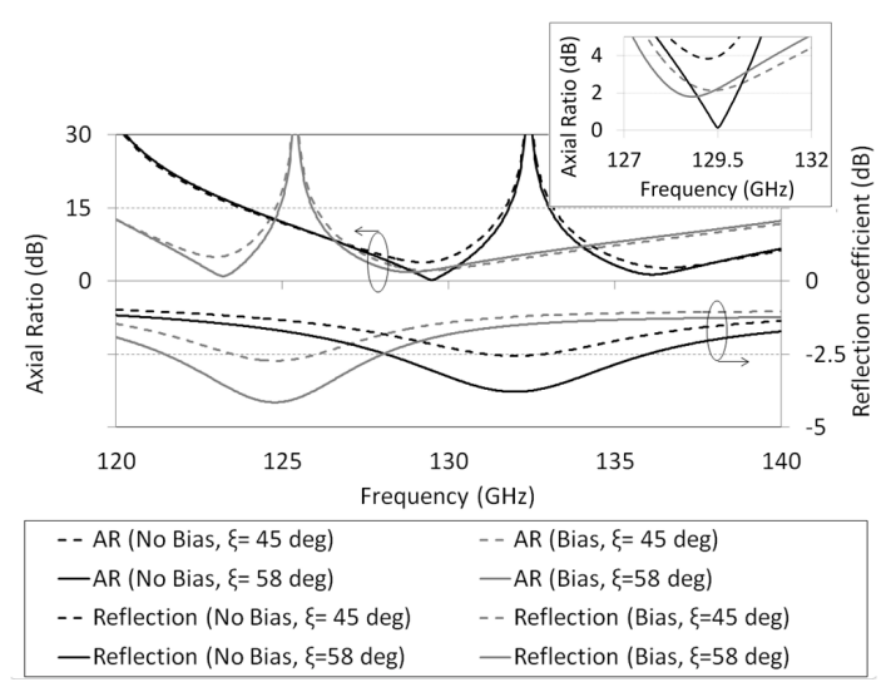

Fig. 4. Simulated axial ratio and reflection magnitudes (obtained with CST) for incoming plane waves at angle $\theta=45^{\circ}$ linearly polarized at angle $\xi=$ $45^{\circ}$ and $\xi=58^{\circ}$ on the unbiased and biased LC based polarizer of Fig. 2. Inset shows axial ratio curves zoomed in the vicinity of the operating frequency.

Using the predicted responses shown in Fig. 3, the axial ratio (AR) of the reflected waves based on an incoming linearly polarized signal at slant angle $\xi=45^{\circ}$ (as defined in Fig. 1) was calculated for both the unbiased and biased states and is plotted in Fig. 4. For each state the AR curve exhibits two minima, which correspond to RHCP and LHCP modes, and a maximum, which corresponds to the orthogonal linear polarization. In particular, Fig. 4 shows that when the polarizer is biased, the AR curve shifts towards lower frequencies. For the unbiased case the phase difference between $\mathrm{TE}$ and $\mathrm{TM}$ waves at $130 \mathrm{GHz}$ is $90^{\circ}$ indicating that reflection occurs in RHCP. For the biased case the next AR minimum, which corresponds to a phase difference between 
TE and TM waves of $270^{\circ}$ and LHCP polarization state, occurs at $128.5 \mathrm{GHz}$; although in principle it would be sufficient for the theoretical design to produce a frequency shift of the AR curve reduced by $1.5 \mathrm{GHz}$ (so that at the biased state LHCP occurs at $130 \mathrm{GHz}$ ), an additional margin has been accounted for in view of experimental uncertainties. Between these two states (intermediate bias) the orthogonal linearly polarized state occurs upon reflection when the AR value exhibits a pole. Hence for this design the change in the permittivity $\left(\varepsilon_{r \perp} \rightarrow \varepsilon_{r / /}\right)$ of the liquid crystals enables the entire meridian of the Poincare sphere to be covered.

Assuming that the surface is illuminated by a plane wave linearly polarized at slant angle $\xi$, the magnitude of the reflection coefficient, $R_{T}$, can be calculated from the magnitude of the measured reflection coefficients for TE and TM incident waves, $R_{T E}, R_{T M}$ respectively and the slant angle $\xi$, using:

$$
R_{T}=10 \cdot \log \left\{\left[R_{T E} \sin (\xi)\right]^{2}+\left[R_{T M} \cos (\xi)\right]^{2}\right\}
$$

Fig. 4 also shows the magnitude of the reflection coefficients for the same orientation angle of the incident signal. The lowest value is approximately $3 \mathrm{~dB}$ less than that shown for TE incidence in Fig. $3 b$; this is because for $\xi=45^{\circ}$ the wave vector can be resolved equally between the TM and TE reflection coefficients of Fig. 3b.

These results in Fig. 4 show that although the phase difference between the TE and TM reflected waves is exactly $90^{\circ}$ at about $130 \mathrm{GHz}$ (Fig. 3a) for the unbiased state, the axial ratio of the RHCP signal is more than $3.8 \mathrm{~dB}$. This is due to the magnitude imbalance between TE and TM reflected waves associated with the increased absorption of the former (Fig. $3 b)$. One option available to compensate for the magnitude of the imbalance between the TE and TM reflection coefficients, and hence reduce the AR of the reflected waves, is to vary the slant angle $\xi$.

According to Fig. 1, the magnitudes $I_{T E}, I_{T M}$ of the TE and TM components at incidence are

$$
I_{T E}=I \cdot \sin (\xi) \quad I_{T M}=I \cdot \cos (\xi)
$$

where $I$ is the magnitude of the incident wave. By increasing $\xi$ it is therefore possible to increase the amplitude of the impinging TE wave so that upon reflection the desired amplitude balance is achieved. Since such a modification does not affect the phase of the reflected TE and TM waves, this approach can be used to improve the polarization purity of the CP signal [23]. The computed AR for slant angle $\xi=58^{\circ}$, which provides an optimum trade-off in terms of AR, tunability and insertion losses, is superimposed on Fig. 4, where values of about $0.5 \mathrm{~dB}$ are now observed for RHCP (unbiased state) at $130 \mathrm{GHz}$. This improvement comes at a cost of a very slight reduction in the polarization purity of the orthogonal $\mathrm{CP}$ signal because in this case the amplitude of the reflected TE component is higher than the TM signal (Fig. $3 \mathrm{~b}$ ), leading to a $0.08 \mathrm{~dB}$ increase in the value of the AR. Likewise, higher values of $\xi$ lead to increased thermal losses because of the increase in the TE absorption. This is depicted in Fig. 4 where the absorption loss for the unbiased case now peaks at $-3.7 \mathrm{~dB}$.

\section{B. Experimental Testing}

The reflection amplitude and phase response of the electronically tunable polarizer prototype were measured using an $\mathrm{AB}$ mm vector network analyzer [24] in conjunction with a quasi-optical test bench. The LC filled polarizer was located at the beam waist in the quasi-optical feed train and the horn pairs were orientated to measure the responses in the TE and TM planes. The beam-waist radius at the location of device under test is approximately $9 \mathrm{~mm}$ and the projected area of the periodic array ( $75 \mathrm{~mm}$ diameter), which is orientated at $45^{\circ}$, is more than four times this value, therefore power loss and diffraction effects due to beam truncation can be neglected. The incident wave front can therefore be considered as a plane wave of infinite transverse extent. A photograph of the measured prototype mounted on the quasioptical test bench is shown in Fig. 7. The reflection response of the polarizer was measured by ratioing the detected reflected power relative to a flat aluminum plate inserted in the sample holder in the position of the polarizer's ground plane. Initially a base level frequency sweep was made followed by a sweep with the device inserted, with the ratio of these giving the spectral characteristics of the test sample. Measurements were made over the frequency range 122-136 $\mathrm{GHz}$ using a large sweep setup, which requires retuning of the system about every $10 \mathrm{GHz}$.

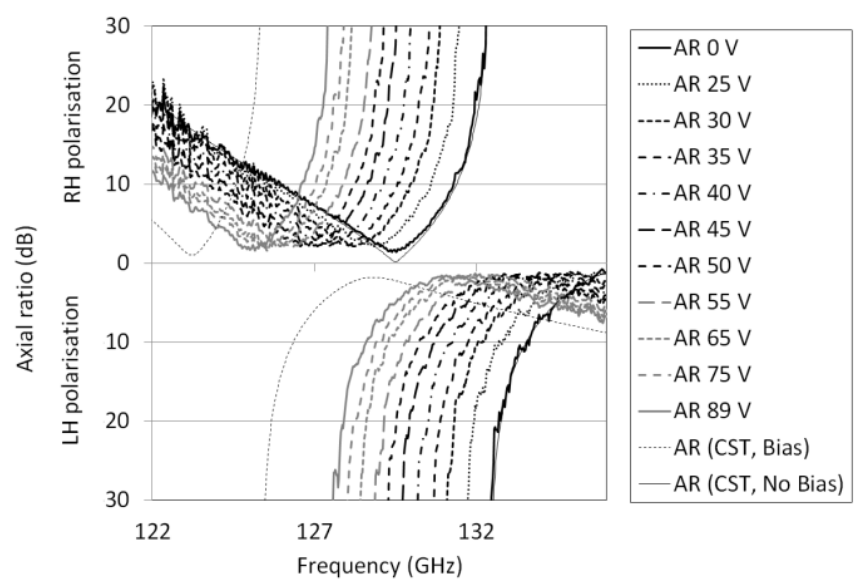

Fig. 5. Measured axial ratio as a function of frequency for different biasing voltage values. The tensors shown in eq. (1) have been used for the simulation of the unbiased (OFF) and biased (ON) states, where $\varepsilon_{/ /}=3.25, \tan \delta_{/ /}=$ $0.015, \varepsilon_{\perp}=2.47, \tan \delta_{\perp}=0.02$. Top half of the plot corresponds to RHCP and lower half of the plot to LHCP. Incoming wave linearly polarized at angle $\xi=58^{\circ}$.

The polarizer was biased using an $\mathrm{AC}$ source operating at $10 \mathrm{~Hz}$ and with adjustable amplitude up to $89 \mathrm{~V}$. In order to compare the measured results with those predicted by fullwave CST simulations, the reflection phase and magnitude measured for $0 \mathrm{~V}, 40 \mathrm{~V}$ and $89 \mathrm{~V}$ are plotted against the simulated results for the biased and unbiased liquid crystal in Fig. 3. The good agreement between the simulated and 
measured TE and TM plane reflection phases for the unbiased state (Fig. 3a) confirms the fabrication accuracy of the LC based polarizer. The measured TM reflection coefficients agree well with the predicted values (Fig. 3b). However the measured TE reflection magnitudes are more than $2 \mathrm{~dB}$ lower than the simulated values. Given that the dielectric stack interacts similarly for both TE and TM incident waves, this is an indication that higher than predicted Ohmic losses occur on the dipole array where strong currents are excited for TE incidence only. It can further be observed that although the simulated spectral shift for the TE AMC frequency between biased and unbiased LC states is over $6.5 \mathrm{GHz}$, the measured shift is only of the order of $4 \mathrm{GHz}$; this could be attributed to the biasing voltage values being limited by the measurement equipment so that the liquid crystal does not reach its fully biased state.

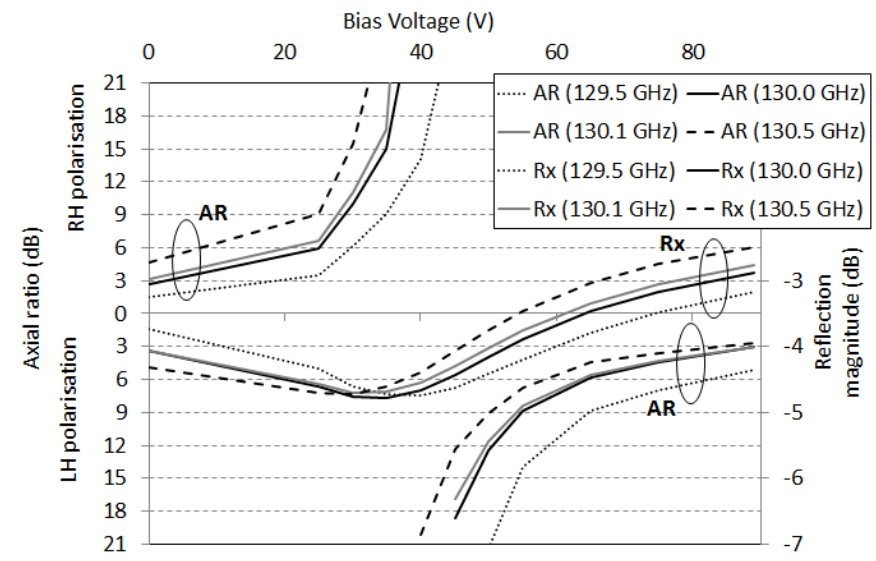

Fig. 6. Detailed view of the measured axial ratio and reflection coefficient magnitude near the operating frequency. Top half of the plot corresponds to RHCP polarization and lower half of the plot to LHCP.

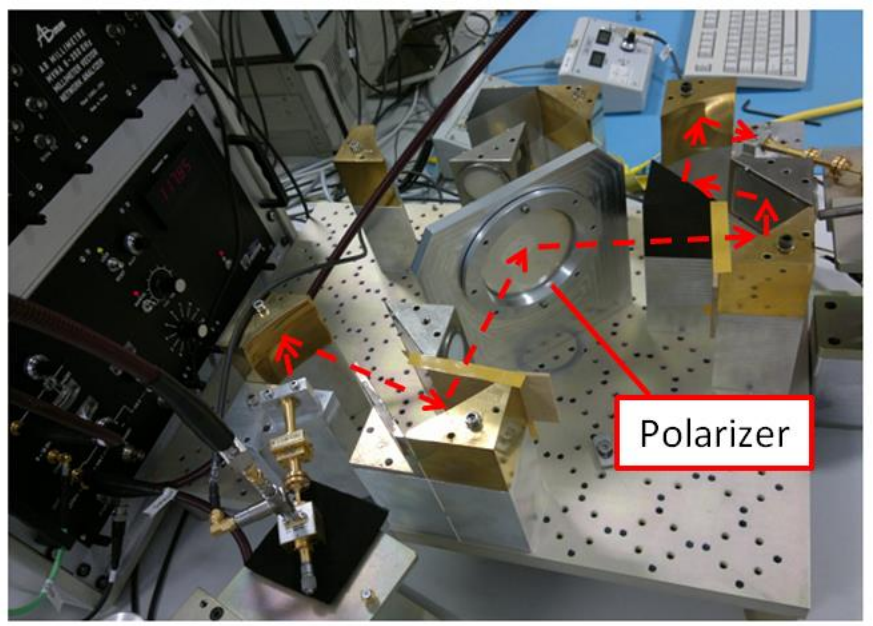

Fig. 7. Photograph of the LC tunable polarizer on the quasi-optical test bench used for measuring reflection properties. A flat metallic plate located at the polarizer's ground plane was employed for calibration.

The axial ratio of the polarizer was calculated for slant angle $\xi=58^{\circ}$ using the measured data for increasingly higher values of the biasing voltage amplitude. This is plotted in Fig. 5 and superimposed with the simulated AR for the biased and unbiased states for comparison. In this plot we have used the top half to represent axial ratio for right-handed polarization and the lower half for left-handed polarization assuming incidence wave polarized at slant angle $\xi=+58^{\circ}$ (the dual is valid for incidence wave polarized at slant $\xi=-58^{\circ}$ ). As shown, for each biasing voltage value there is a frequency point with axial ratio values in excess of $30 \mathrm{~dB}$; this corresponds to reflection of a wave linear polarized at an angle supplementary to that of the incidence signal. At lower frequencies, the axial ratio approaches the $3 \mathrm{~dB}$ RHCP while at higher frequencies the axial ratio approaches the $3 \mathrm{~dB}$ LHCP. In accordance to Fig. 3, good agreement between the measured and simulated AR for the unbiased case is obtained with the measured minimum AR value compromised to a minimum level of about $1.6 \mathrm{~dB}$ due to the amplitude imbalance. Likewise the discrepancy between the simulated AR for the biased state and the measured AR for $89 \mathrm{~V}$ is attributed to the limited biasing voltage.

In order to probe further on the performance of the polarizer at and in the vicinity of the operating frequency, Fig. 6 plots the axial ratio and reflection coefficient magnitude calculated as above against the bias voltage. As shown, at $130 \mathrm{GHz}$, the reflected wave is RHCP with an axial ratio of $3 \mathrm{~dB}$ for $0 \mathrm{~V}$ bias voltage and LHCP with an axial ratio of $3 \mathrm{~dB}$ for $89 \mathrm{~V}$ bias voltage. For $40 \mathrm{~V}$ bias voltage, the reflected wave is linearly polarized at $\xi=-58^{\circ}$ with polarization purity in excess of $30 \mathrm{~dB}$. This performance is largely maintained within a bandwidth of about $1 \mathrm{GHz}$; at frequencies different than the nominal value of $130 \mathrm{GHz}$, the polarization purity of either RHCP/LHCP increases at the expense of the LHCP/RHCP state.

\section{DISCUSSION AND CONCLUSION}

An electronically tunable reflection polarizer based on liquid crystal substrate has been proposed for $\mathrm{mm}$-wave and $\mathrm{THz}$ applications. It is noted that beyond reflection polarizers, the proposed concept provides a pertinent solution for electronically reconfigurable phase plates; an observation of Fig. 4a shows that for one linear polarization (TE) it is possible to adjust the reflection phase over a range in excess of $180^{\circ}$. By exploiting the scattering properties of arrays with polarization independent responses [25], it is possible to provide identical reflection magnitude and phase coefficients for all incoming wave polarizations. Therefore with the exception of the unavoidable increased thermal losses, such structures are the electronically reconfigurable equivalent of a mechanically moving flat metallic reflector that can shift along the direction of an incoming beam by an adjustable distance of up to half wavelength. Arbitrary electrical length ranges can be achieved if the phase agile mirror is designed to provide reflection phases over a range of $360^{\circ}$. These surfaces can thus find application in phase retrieval for $\mathrm{mm}$-wave and THz radars [26] or tunable interferometers [6].

\section{ACKNOWLEDGMENT}

This work was supported by The European Space Agency (grant A0/1-6169/09/NL/JD). The authors are grateful to $\mathrm{Mr}$ Atsutaka Manabe of Merck KGaA, Darmstadt, Germany for 
fruitful discussions and for supplying the liquid crystal materials.

\section{REFERENCES}

[1] WindSat radiomenter, Naval Research Laboratory Remote Sensing Division, http://www.nrl.navy.mil/WindSat/, accessed on 12 August 2013

[2] P. W. Gaiser, etal, "The WindSat spaceborne polarimetric microwave radiometer: Sensor description and early orbit performance", IEEE Trans. Geosc. \& Remote Sensing, vol. 42, no. 11, pp. 2347-2361, Nov. 2004.

[3] European Space Agency, Polarimetric SAR data processing and education tool, http://earth.eo.esa.int/polsarpro/tutorial.html, accessed on 12 August 2013

[4] K. Sarabandi, "A waveguide polarization controller," IEEE Trans. Microw. Theory Techn., vol. 42, No. 11, pp. 217-21741, Nov. 1994

[5] S. Hollung, W. A. Shiroma, M. Markovic, and Z. B. Popovic, "A quasioptical isolator," IEEE Microw. Guided Wave Lett., vol. 6, no. 5, pp. 205-206, May 1996.

[6] T. Manabe, J. Inatani, A. Murk, R. J. Wylde, M. Seta and D. H. Martin, "A new configuration of polarization-rotating dual-beam interferometer for space use," IEEE Trans. Microw. Theory and Techn., vol. 51, no. 6, pp. 1696-1704, Jun. 2003.

[7] J.A. Ruiz-Cruz, M.M. Fahmi, M. Daneshmand, R.R Mansour, "Compact reconfigurable waveguide circular polarizer," IEEE Microwave Symposium Digest, June 2011.

[8] J.A. Ruiz-Cruz, M.M. Fahmi, S.A. Fouladi, R.R. Mansour, "Waveguide antenna feeders with integrated reconfigurable dual circular polarization," IEEE Trans. Microwave Theory Techn., vol. 59, no. 12, pp. 3365-3374, December 2011.

[9] W. Hu, R. Cahill, J. A. Encinar, R. Dickie, H. S. Gamble, V. Fusco, and $\mathrm{N}$. Grant, "Design and measurement of reconfigurable $\mathrm{mm}$ wave reflectarray cells with nematic Liquid Crystal," IEEE Trans. Antennas Propag., vol. 56, no. 10, pp. 3112- 3117, Oct. 2008.

[10] W. Hu, M. Y. Ismail, R. Cahill, H. S. Gamble, R. Dickie, V. F. Fusco, D. Linton, S. P. Rea, and N. Grant, N, "Tunable liquid crystal reflectarray patch element," Electron. Lett., vol.42, no.9, pp. 15- 16, April, 2006.

[11] A. Moessinger, R. Marin, S. Muller, J. Frees, and R. Jakoby, "Electronically reconfigurable reflectarrays with nematic liquid crystals," Electron. Lett., vol.42, no.9, pp 899-890, August 2006.

[12] S. Strunck, O.H. Karabey, A. Gaebler and R. Jakoby, "Reconfigurable waveguide polariser based on liquid crystal for continuous tuning of linear polarisation," Electron. Lett., vol. 48, no. 8, pp 441-443, April 2012.

[13] W. Hu, R. Dickie, R. Cahill, H. S. Gamble, M. Y. Ismail, V. Fusco, D. Linton, S. P. Rea and N. Grant, "Liquid crystal tunable $\mathrm{mm}$ wave frequency selective surface," IEEE Microw. and Wirel. Comp. Lett., vol. 17, no. 9, pp. 667- 700, Sept. 2007.

[14] W. Hu, M. Y. Ismail, R. Cahill, J. A. Encinar, V. F. Fusco, H. S. Gamble, D. Linton, R. Dickie, N. Grant and S. P. Rea, "Liquid crystal based reflectarray antenna with electronically switchable monopulse patterns," Electron. Lett., vol. 43, no.14, pp. 744 -745, July, 2007.

[15] W. Hu, M. Arrebola, R. Cahill, J. A. Encinar, H. S. Gamble, V. Fusco, Y. Alvarez, and F. Las-Heras, "94 GHz Beam Steering Dual Reflector Antenna with Reflectarray Subreflector," Proc IEEE Antennas \& Propag., vol. 57, no.10, pp., 3043 - 3050, Oct. 2009.

[16] E. Doumanis, G. Goussetis, J. L. Gomez-Tornero, R. Cahill, V. Fusco, "Anisotropic Impedance Surfaces for Linear to Circular Polarization Conversion," IEEE Trans. Antennas \& Propag., vol. 60, Iss. 1, pp. 212219, Jan. 2012.

[17] E. Doumanis, G. Goussetis, J. L. Gomez-Tornero, R. Cahill, V. Fusco, "Mm-wave low-profile reflection polarizer," Proc. Microw. Workshop On Millimeter Wave Integration Technologies (IMWS), pp. 180-183, Sept, 2011.

[18] CST Microwave Studio. [Online]. Available: http://www.cst.com.

[19] R. Dickie, P. Baine, R. Cahill, E. Doumanis, G. Goussetis, S. Christie, N. Mitchell, V. Fusco, J. A. Encinar, R. Dudley, D. Hindley, M. Naftaly, M. Arrebola and G. Toso, "Electrical Characterisation of Liquid Crystals at $\mathrm{mm}$ wavelengths using frequency selective surfaces," IET Electron. Lett., vol. 48, no. 11, pp. 611 -612, May, 2012.

[20] [Online]. Available: http://sekisui-corp.com/prev/spd/product pages /fc/html/lcd/lcd_01.html
[21] Perez-Palomino, P. Baine, R. Dickie, M. Bain, J. A. Encinar, R. Cahill, M. Barba, and G. Toso, "Design and Experimental Validation of Liquid Crystal-Based Reconfigurable Reflectarray Elements With Improved Bandwidth in F-Band," IEEE Trans. Antennas \& Propag., vol. 61, no. 4, pp. 1704-1713, Apr. 2013.

[22] G. Goussetis, A.P. Feresidis, J.C. Vardaxoglou, "Tailoring the AMC and EBG Characteristics of Periodic Metallic Arrays Printed on Grounded on Grounded Dielectric Substrate," IEEE Transactions Antennas and Propagation, Vol. 54, No. 1, pp. 82-89, January 2006

[23] B. Toh, R. Cahill, V. Fusco, "Understanding and measuring circular polarization," IEEE Trans. Education, Vol. 46, No. 3, pp. 313-319, Aug. 2003

[24] AB Millimetre, [Online]. Available: http://www.abmillimetre.com

[25] R. Dickie, R. Cahill, H. Gamble, V. Fusco, M. Henry, M. Oldfield, P. Huggard, P. Howard, N. Grant, Y. Munro, P. de Maagt, "Submillimeter Wave Frequency Selective Surface With Polarization Independent Spectral Responses," IEEE Trans. Antennas Propag., Vol. 57, No. 7, pp. 1985 - 1994, 2009

[26] G. Hislop, L. Li, A. Hellicar, "Phase Retrieval for Millimeter- and Submillimeter-Wave Imaging," IEEE Trans. Antennas Propagation, Vol. 57, No. 1, pp. 286-290. Jan. 2009 